\title{
Frequency and anatomic variability of the mandibular lingual foramina: a cone-beam CT study
}

Silvio Taschieri ${ }^{1,2,3}$, Stefano Corbella ${ }^{1,2,3}$, Amel Silnovic ${ }^{1}$, Luca Francetti ${ }^{1,2}$, Carmelo Messina ${ }^{2}$, Luca Maria Sconfienza ${ }^{2,4}$ and Domenico Albano ${ }^{2,5^{*}}$ (i)

\begin{abstract}
Background: To evaluate the distribution of lingual foramina (LF) and their correlation with demographic characteristics and mandible width, shape, and bone thickness in Caucasian Italian patients subjected to cone-beam CT (CBCT).
\end{abstract}

Methods: CBCTs were reviewed to assess the number of all LF, midline and lateral LF. We also assessed the relationship of the number of lateral LF with gender and mandibular width, shape, and bone thickness using the Chi Square test. A $p$ value $<0.05$ was considered statistically significant.

Results: Three-hundred patients (180 males; age range: $21-87$ years) were included. The highest frequency per patient was of 2 LF $(97 / 300,32.3 \%)$, followed by $3(81 / 300,27 \%)$ and 4 (53/300, 17.7\%). No LF were observed in 2/300 patients $(0.7 \%)$, while the highest number was of $8 \mathrm{LF}$ in one patient. The highest frequency of midline LF per person was of $2 \mathrm{LF}(57.3 \%, 172 / 300)$, while the highest number per person was $5 \mathrm{LF}$ in one patient $(0.3 \%)$. The highest frequency of midline LF located above and below the genial tubercle was of 1 in 197/300 patients (65.7\%) and in $169 / 300$ patients (56.3\%), respectively. Concerning lateral $L F$, the highest frequencies were of $0(113 / 300,37.7 \%)$ and of $1(112 / 300,37.3 \%)$. We did not observe any significant difference of the number of midline and lateral LF based on gender ( $P=.438$ and $P=.195$, respectively) or mandible width ( $P=.069$ and $P=.114$, respectively). The mandible shape was normal in 188 cases, with facial constriction in 42, lingual constriction in 54 , and hour glass constriction in 16. The mean bone thickness was $10.76 \mathrm{~mm}$ in the symphysis, $10.92 \mathrm{~mm}$ in the right hemiarches, and $10.68 \mathrm{in}$ the left hemiarches. No significant differences in the distribution of LF were observed also based on mandibular shape and bone thickness (both with $P>$.05).

Conclusions: We have shown the high variability of number and anatomic distribution of LF in an Italian group of patients subjected to CBCT without reporting any association with gender and mandible width, shape, and bone thickness.

Keywords: Cone-beam computed tomography, Lingual foramen, Mandible, Anatomy, Vascularization

*Correspondence: albanodomenico@me.com

${ }^{2}$ IRCCS Istituto Ortopedico Galeazzi, Milan, Italy

Full list of author information is available at the end of the article

\section{Background}

The lingual foramina (LF) host important vascular and neural structures coming from the floor of mouth that perforate the cortical bone of the mandible on the lingual side providing vascular and nerve supply to the mental region [1]. Specifically, the sublingual artery, which is a 
branch of the lingual artery, travels along the superior face of the mylohyoid muscle and then through the LF and anastomoses with central inferior alveolar vessels, while the submental artery, which is a branch of the facial artery, travels along the inferior face of the mylohyoid muscle and then penetrates the mental region to anastomose with branches of the anterior alveolar artery [2, 3]. An extreme variability has been reported in the number and topographic distribution of LF and in the type and number of anastomoses between these two arteries. Despite the complex vascularization of the floor of mouth and mental region, there is a diffuse mistaken belief among oral surgeons that the mandibular region included between the mental foramina is the safest for surgery procedures. Unfortunately, the proof of this misconception is the non-negligible number of massive bleeding accidents having been reported after implant interventions in this region [4-8]. Such cases, even though not so frequent as compared to the total number of implants placed, could be a significant life hazard. For such reason, a detailed anatomic knowledge of the vascularization of the interforaminal area as well as a thorough pre-operative imaging evaluation of the variability of LF are essential to prevent life-threatening complications during surgical procedures. Indeed, the individual anatomical variability of mandibular neuro-vascular bundles is a crucial factor to be considered when dealing with mandibular surgery [9]. Previous cadaveric and imaging studies, mostly based on computed tomography (CT) examinations, have investigated this point highlighting the extreme anatomic variability between different populations [10]. The aim our study was to evaluate the number and position of LF and to evaluate if correlation exists with demographic characteristics and mandible width, shape, and bone thickness in a sample made of Caucasian Italian patients subjected to cone-beam CT (CBCT).

\section{Methods}

This retrospective study was approved by our Institutional Review Board and the requirement for informed consent was waived. After matching imaging and demographic data, our dataset was anonymized to remove any connections between data and patients' identity according to the General Data Protection Regulation for Research Hospitals.

\section{Patient population}

This study is concerned with the evaluation of anatomic variability of LF in a consecutive series of Caucasian Italian patients subjected to CBCT at our Institution from January 2017 to December 2020. CBCT scans were done for several reasons, including pre-operative planning for implant or dental surgery. We included in our series all
Caucasian Italian patients with permanent (non-mixed or deciduous) teeth with age $\geq 20$ years. The following exclusion criteria were applied: (i) edentulism, specifically the absence of mandibular central/lateral incisors and canines, since mandible morphology may vary substantially in patients with this condition; (ii) motion artifacts such that compromise images quality making not possible the evaluation of LF; (iii) previous mandibular surgery like osteotomy; (iv) bony lesions of the mandible; (v) mandibular fractures.

\section{$\mathrm{CBCT}$ and images interpretation}

CBCT scans were done on a 3D Accuitomo XYZ Slice View Tomograph ${ }^{\circledR}$ (Model MCT-1, Type EX-1/EX-2; Fushimi-ku, Kyoto: J. Morita Mfg. Corp). The acquisition protocol included the following imaging parameters: tube voltage, $60-80 \mathrm{kV}$; tube current, $1-10 \mathrm{~mA}$; a voxel size of $0.125 \times 0.125 \times 0.125 \mathrm{~mm}$. First, the measurements were taken by an undergraduate dentistry student with 4 years of experience in $\mathrm{CBCT}$ imaging, previously calibrated with a board-certified experienced radiologist (D.A.), with 8 years of experience in CBCT imaging, by examining together 30 sample scans, until reaching a concordance in measurements. Both reviewers were blinded with patients age and gender. Even after calibration, the entire process of images review was supervised by a radiologist (D.A.), but only one final set of measurements were obtained by the undergraduate dentistry student. Notably, the high reproducibility of these measurements has been already proven by previous studies [11, 12]. We have reviewed all images in a liquid crystal display monitor backlit with cold-cathode fluorescent lamps.

All scans were evaluated to assess:

- the number of all LF, including both midline and lateral foramina (Fig. 1);

- the number and position of midline LF in the middle point of the symphysis differentiating those located above the genial tubercle (generally considered as the foramen of the sublingual artery) from those located below it (generally considered as the foramen of the submental artery) (Fig. 2);

- the number and position of lateral foramina, dividing them in four groups on the basis of the relationship with the teeth (incisors, canines, premolars, and molars) (Fig. 3);

- mandibular shape, given that the mandible cross section can be either normal, or with facial constriction, lingual constriction, and hour glass constriction [13];

- mandibular bone thickness measured in the symphysis and in both hemiarches at the level of the first molars (Fig. 4). 


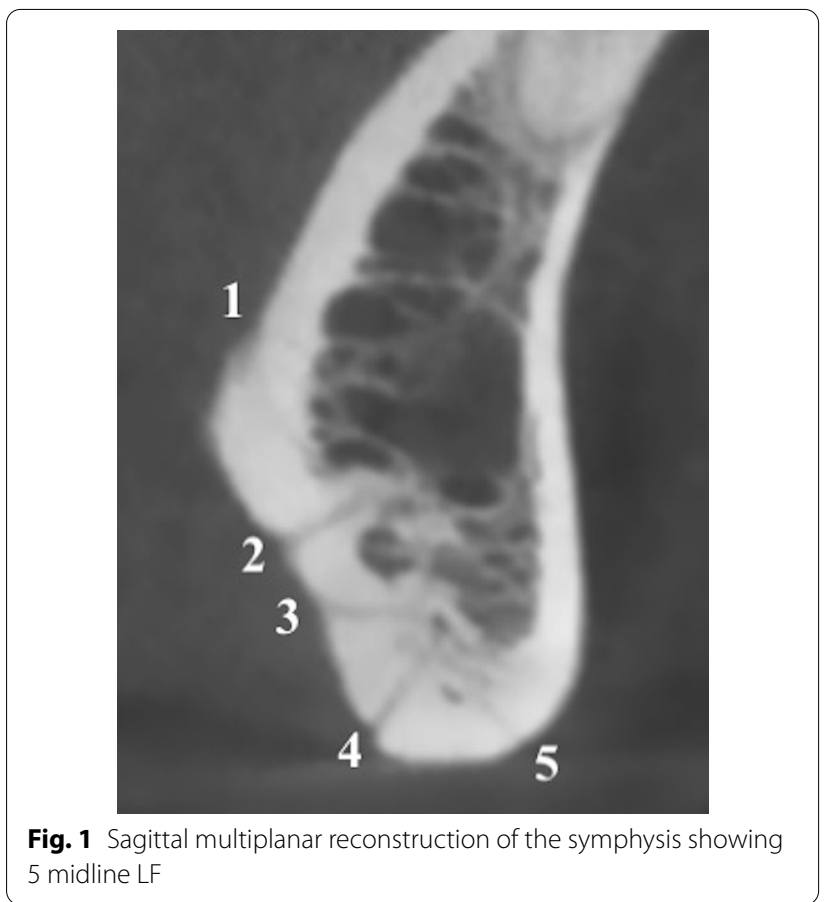

To assess the relationship existing between mandibular width and number/position of lateral foramina, we have estimated the area of the floor of mouth considering it as a triangle. To do that, we have calculated the distance between the mental foramina (base of the triangle) on the multiplanar axial reconstruction obtained to see in the

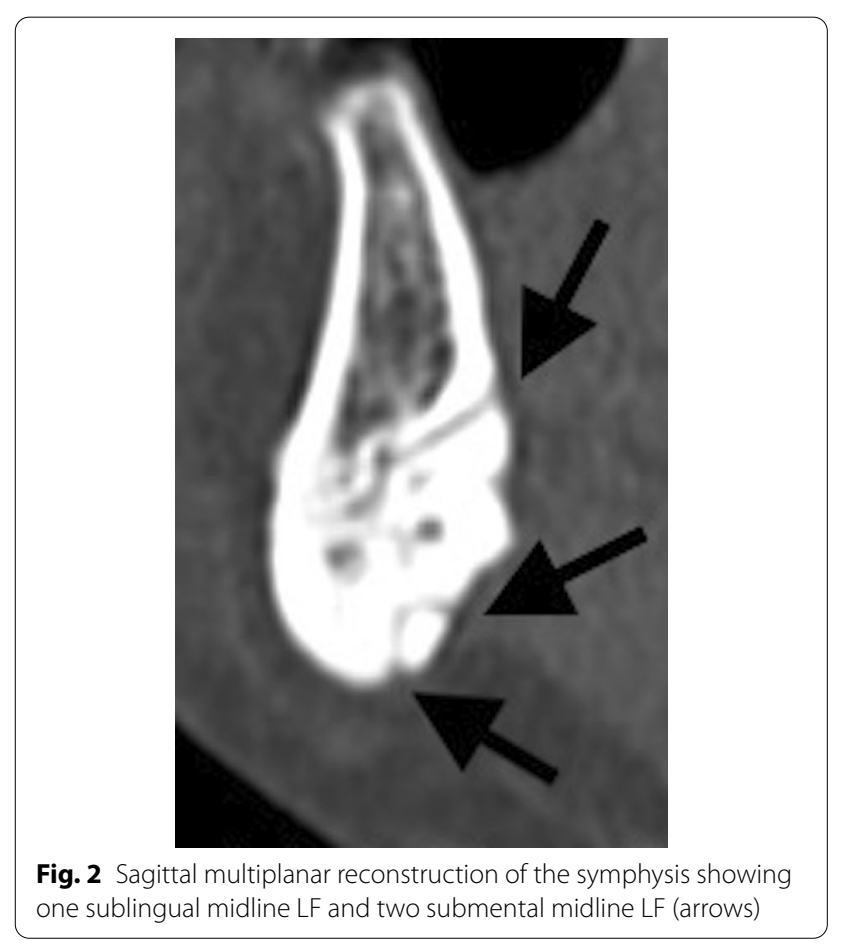

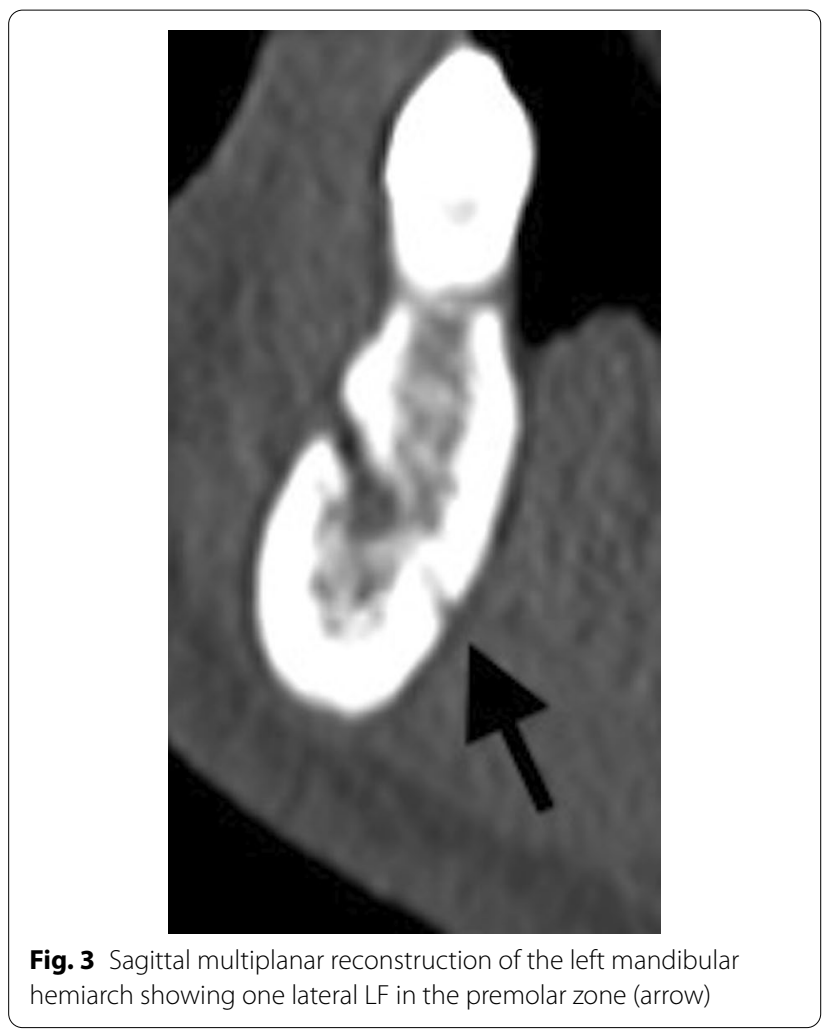

same slice both foramina and we have traced a perpendicular line passing reaching the symphysis (height of the triangle). The estimated area of the floor of mouth was then calculated with the following formula as shown in Fig. 5:

$\frac{\text { base } \times \text { height of the triangle }}{2}$

The estimated area of the floor of mouth ranged from 17.27 to $30.13 \mathrm{~cm}^{2}$. To correlate the evaluation of LF to the width of the mandible, we divided the patients in three groups of 100 on the basis of the estimated area of the floor of mouth: between $17.27 \mathrm{~cm}^{2}$ and $22.47 \mathrm{~cm}^{2}$

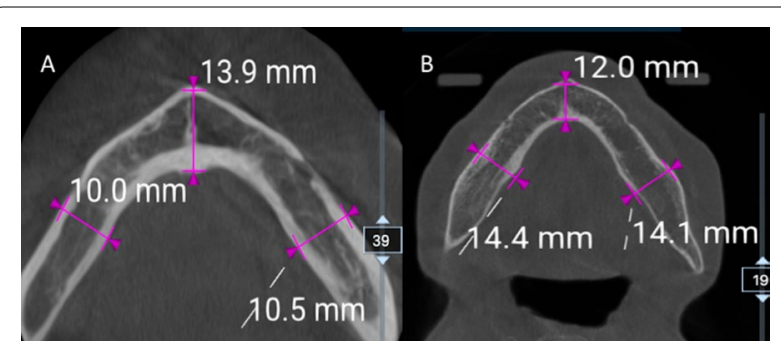

Fig. 4 Axial CBCT images of two different patients ( $\mathbf{A}$ and $\mathbf{B}$ ) with mandibular bone thickness measured in the symphysis and in both hemiarches at the level of the first molars 
(Group1), between 22.52 and $24.56 \mathrm{~cm}^{2}$ (Group2), and between 24.57 and $30.13 \mathrm{~cm}^{2}$ (Group3).

\section{Statistical analysis}

We compared the frequency of midline and lateral LF on the basis of gender, mandibular width, shape, and bone thickness using the Chi Square test. For categorical variables, frequencies were provided. Statistical analysis was performed using SPSS ${ }^{\circledR}$ software (v. 26, IBM, Armonk, New York, NY). A $p$ value $<0.05$ was considered as statistically significant.

\section{Results}

According to our criteria, 300 Caucasian Italian patients (180 males, 120 females; age range 21-87 years) were included in this analysis. The highest frequency per patient observed in our series was of $2 \mathrm{LF}(97 / 300,32.3 \%)$, followed by $3(81 / 300,27 \%)$ and $4(53 / 300,17.7 \%)$. No LF were observed in $2 / 300$ patients $(0.7 \%)$, while the highest number of LF $(n=8)$ was observed in only one patient $(0.3 \%)$. Regarding midline LF, the highest frequency per patient was of $2 \operatorname{LF}(57.3 \%, 172 / 300)$, while the highest number per patient was of $5 \mathrm{LF}$ in just one patient $(0.3 \%)$ (Fig. 1). The highest frequency of midline LF located above and below the genial tubercle was of 1 in 197/300 patients $(65.7 \%)$ and in $169 / 300$ patients $(56.3 \%)$, respectively. Then, concerning lateral LF, the highest frequencies were of $0(113 / 300,37.7 \%)$ and of $1(112 / 300,37.3 \%)$. We did not observe any statistically significant difference of the number of midline and lateral LF based on gender ( $P=0.438$ and $P=0.195$, respectively) or mandible width ( $P=0.069$ and $P=0.114$, respectively). Data concerning the total number and topographic distribution of LF is reported in Table 1, while full data stratified for the mandible width and the relationship of LF with the teeth is reported in Table 2.

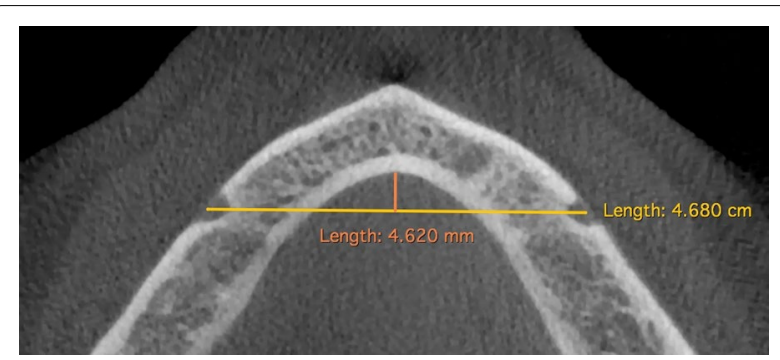

Fig. 5 Axial multiplanar reconstruction to estimate the area of the floor of mouth considering it as a triangle. The distance between the mental foramina (base of the triangle) was $4.680 \mathrm{~cm}$ and the perpendicular line reaching the symphysis (height of the triangle) was $0.462 \mathrm{~cm}$. The estimated area of the floor of mouth was then calculated obtaining $25.69 \mathrm{~cm}^{2}$
The mandible shape was normal in 188 cases, with facial constriction in 42, lingual constriction in 54, and hour glass constriction in 16 . The mean bone thickness was $10.76 \mathrm{~mm}$ in the symphysis, $10.92 \mathrm{~mm}$ in the right hemiarches, and 10.68 in the left hemiarches. No significant differences in the distribution of LF were observed based on mandibular shape and bone thickness (both with $P>0.05$ ).

\section{Discussion}

Our main finding is the extreme variability of the number and topographic distribution in midline and lateral LF. The first consideration to be pointed out is that the absence of LF is quite rare but possible, despite several studies have reported at least one LF in their series. It could be postulated that we wrongly missed the LF in those two patients of our series, but also Sekerci et al. [11] and Demiralp et al. [12] found few patients without LF in their series, in $1.8 \%$ and $3.4 \%$ respectively, thus supporting our results. Concerning the maximum number of LF per patient, we found up to seven LF in one out of 300 patients and eight LF in another patient. Indeed, a large number of LF can be rarely encountered as highlighted by previous studies. Similarly, He et al. found up to seven LF in $2 \%$ of their patients [14]. Notably, Patil et al. reported up to $11 \mathrm{LF}$ in $0.5 \%$ of their CT study on 300 patients [15]. However, the total number of LF per patient is generally quite lower. In fact, in our series, two LF were observed in $32.3 \%$ of patients, three in $27 \%$, and four in $17.7 \%$. These results are in line with those previously published, given that two LF were reported in $28.2 \%$ of patients by Sekerci et al. in $34 \%$ by Patil et al., and in $32.8 \%$ by Demiralp $[11,12,15]$. Conversely, higher variability has been reported concerning the frequency of patients with only one LF that was $10 \%$ in our series, similarly to what reported by He et al. (12\%) and Demiralp et al. $(10.3 \%)[12,14]$, but quite lower than in other series in which one LF has been observed as the most common rate of $L F$ (in up to $40 \%$ patients) $[15,16]$.

Concerning the midline LF, two previous studies confirmed the chance of having no LF in the middle portion of the symphysis with frequencies of $3-3.8 \%$ [17, 18]. Nevertheless, no other studies reported more than four LF in the midline, although we found five LF in one patient. We also found four LF in 4/300 patients (1.3\%), which is a rare but possible condition also reported by Sheikhi et al. and Wang et al. in $2.9 \%$ and $2 \%$ of cases, respectively $[17,19]$. Further, we reported the highest frequency of patients presenting two midline LF ever published (in $57.3 \%$ of cases). Of note, two LF has been the highest frequency of midline LF also in several previous studies, having been observed in $53.9 \%$ by Von Arx et al., in $52.9 \%$ by Sheikhi et al., in $43.6 \%$ by Wang et al., and in 
Table 1 Data concerning the total number and topographic distribution of midline and lateral LF

\section{Total LF}

0: 2/300 (0.7\%); 1: $30 / 300$ (10\%); 2: $97 / 300$ (32.3\%); 3: 81/300 (27\%); 4: 53/300 (17.7\%); 5: 29/300 (9.7\%); 6: 6/300 (2\%); 7: 1/300 (0.3\%); $8:$ $1 / 300(0.3 \%)$

\begin{tabular}{|c|c|c|c|c|}
\hline \multicolumn{5}{|l|}{ Midline LF } \\
\hline \multicolumn{2}{|l|}{ Total midline LF } & Sublingual LF & \multicolumn{2}{|l|}{ Submental LF } \\
\hline \multicolumn{2}{|l|}{$0: 2 / 300(0.7 \%)$} & 0: 2/300 (0.7\%) & \multicolumn{2}{|l|}{ 0: 122/300 (40.7\%) } \\
\hline \multicolumn{2}{|l|}{ 1: 68/300 (22.7\%) } & 1: 197/300 (65.7\%) & \multicolumn{2}{|l|}{ 1: 169/300 (56.3\%) } \\
\hline \multicolumn{2}{|l|}{ 2: 172/300 (57.3\%) } & 2: $96 / 300(32 \%)$ & \multicolumn{2}{|l|}{ 2: 9/300 (3\%) } \\
\hline \multicolumn{2}{|l|}{ 3: 53/300 (17.7\%) } & \multicolumn{3}{|l|}{ 3: 4/300 (1.3\%) } \\
\hline \multicolumn{2}{|l|}{ 4: 4/300 (1.3\%) } & \multicolumn{3}{|l|}{ 4: $1 / 300(0.3 \%)$} \\
\hline \multicolumn{5}{|l|}{ 5: 1/300 (0.3\%) } \\
\hline \multicolumn{5}{|l|}{ Lateral LF } \\
\hline Total lateral LF & Incisors & Canines & Premolars & Molars \\
\hline 0: 113/300 (37.7\%) & 0: $261 / 300(87 \%)$ & 0: $271 / 300(90.3 \%)$ & 0: $160 / 300(53.3 \%)$ & 0: $284 / 300(94.7 \%$ \\
\hline 1: 112/300 (37.3\%) & 1: 33/300 (11\%) & 1: 26/300 (8.7\%) & 1: 97/300 (32.3\%) & 1: 14/300 (4.7\%) \\
\hline 2: 59/300(19.7\%) & 2: 6/300 (2\%) & 2: 3/300 (1\%) & 2: $36 / 300(12 \%)$ & 2: $2 / 300(0.7 \%)$ \\
\hline 3: 11/300 (3.7\%) & & & 3: 7/300 (2.3\%) & \\
\hline 4: 5/300 (1.7\%) & & & & \\
\hline
\end{tabular}

Table 2 Data stratified for mandible width and the relationship of LF with the teeth

\begin{tabular}{|c|c|c|c|c|c|c|}
\hline Total LF & Midline LF & Lateral LF & Incisors & Canines & Premolars & Molars \\
\hline \multicolumn{7}{|l|}{ Group 1} \\
\hline 1:11/100 & 1: 31/100 & 0:38/100 & 0: $89 / 100$ & 0: 91/100 & 0:61/100 & 0: 93/100 \\
\hline 2: 40/100 & 2: 52/100 & 1: 47/100 & 1:8/100 & 1:8/100 & 1:32/100 & 1:5/100 \\
\hline 3: 29/100 & 3: 17/100 & 2: 14/100 & 2: $3 / 100$ & 2: $1 / 100$ & 2: 6/100 & 2: $1 / 100$ \\
\hline 4: $15 / 100$ & & 3: 1/100 & & & 3: $1 / 100$ & \\
\hline \multicolumn{7}{|l|}{ 5: 4/100 } \\
\hline \multicolumn{7}{|l|}{ 6: 1/100 } \\
\hline \multicolumn{7}{|l|}{ Group 2} \\
\hline 1: 13/100 & 1: 23/100 & 0:39/100 & 0: $87 / 100$ & 0: 91/100 & 0: 53/100 & 0: 97/100 \\
\hline 2:31/100 & 2: 58/100 & 1:31/100 & 1: 10/100 & 1: 9/100 & 1:27/100 & 1:2/100 \\
\hline 3:22/100 & 3: 16/100 & 2: $24 / 100$ & 2: 3/100 & & 2: $17 / 100$ & 2: $1 / 100$ \\
\hline 4: 20/100 & 4:3/100 & $3: 4 / 100$ & & & 3: 3/100 & \\
\hline 5: 10/100 & & 4: $2 / 100$ & & & & \\
\hline $6: 2 / 100$ & & 5: 0/100 & & & & \\
\hline \multicolumn{7}{|l|}{ 7: 1/100 } \\
\hline \multicolumn{7}{|l|}{ 8: 1/100 } \\
\hline \multicolumn{7}{|l|}{ Group 3} \\
\hline $\mathbf{0 : 2 / 1 0 0}$ & $\mathbf{0 : 2 / 1 0 0}$ & 0:36/100 & 0: $85 / 100$ & 0: $89 / 100$ & 0: 47/100 & 0: 93/100 \\
\hline 1:6/100 & 1: 14/100 & 1:34/100 & 1: 15/100 & 1: 9/100 & $1: 38 / 100$ & $1: 7 / 100$ \\
\hline 2: 26/100 & 2: $62 / 100$ & 2: 21/100 & & 2: $2 / 100$ & 2: $12 / 100$ & \\
\hline 3: 30/100 & 3: 20/100 & $3: 6 / 100$ & & & $3: 3 / 100$ & \\
\hline 4: $18 / 100$ & 4: 1/100 & 4: $3 / 100$ & & & & \\
\hline 5:15/100 & 5: 1/100 & & & & & \\
\hline 6:3/100 & & & & & & \\
\hline
\end{tabular}


$43 \%$ by Rosano et al. [5, 17-19]. As a matter of fact, other studies have reported one LF as the highest frequency of LF in the midline, with frequencies reaching $72 \%$ [20], while one midline LF was observed in $22.7 \%$ of our patients. On the other hand, our frequency of three midline LF $(17.7 \%)$ is in line with previous studies, in which the frequency of three LF per person has never been reported as the most common rate of midline LF. Regarding the relationship with the genial tubercle, we found that a midline LF above the tubercle is almost invariably detected, similarly to what reported by Tagaya et al. $(95 \%)$ and Sheikhi et al. (99\%) $[19,21]$. Further, when a second midline LF is identified, it is located below the genial tubercle in most of the cases. Out of 300 patients, 178 (59.3\%) presented at least one midline LF below the genial tubercle, with a slightly lower frequency to that reported by previous studies with frequency ranging from 74.5 to $85 \%[19,22]$.

The frequency of lateral LF reported in literature is quite variable. We observed lateral LF in $62.3 \%$ of cases, similarly to Liang et al. (62\%) [20], although this frequency was much lower in the series by $\mathrm{He}$ et al. (30.1\%) and higher in that by Tagaya et al. (80\%) [14, 21]. Generally, lateral LF are bilateral and symmetrical, as already reported by previous studies [23]. The total number of LF has been also investigated by Xie et al. who reported one lateral LF in $37.3 \%$, two in $19.7 \%$, three or more in $5,4 \%$ [24], in much the same way of our study (one lateral LF in $36.2 \%$, two in $17.7 \%$, three or more in $0,4 \%$ ). Concerning the relationship between the position of lateral LF and the teeth, we observed $13 \%$ of LF in the zone of incisors, 9.8\% canines, $46.6 \%$ premolars, and $5.4 \%$ molars, confirming what already highlighted by other authors on the highest frequency of lateral LF in the zone of premolars $[11,16,18,24]$. We have resumed in Table 3 the number of LF reported in previous CT and cadaveric studies conducted on different populations.
No previous studies have investigated the association of mandible width, shape, and bone thickness with number and distribution of LF, thus a comparison with the literature is not possible. We did not find any statistically significant association with these mandible measurements, as well with gender, although a progressive increase of the number of lateral LF has been observed from patients with smaller mandible width (first group) presenting 78 LF, to those with bigger mandible width (second group with 99 LF and third group with 106 LF), thus some considerations should be pointed out. In the first group, only $1 \%$ of subjects showed three or more lateral LF, while in the second and third groups the frequency was $6 \%$ and $9 \%$, respectively. Further, considering only the premolars zone, $61 \%$ of patients of the first group did not show any lateral LF, as well as the $53 \%$ of the second group and the $43 \%$ of third.

One of the novelties of this paper is that we attempted to correlate the presence/number of LF to other anatomical aspects, such as the size, shape, and bone thickness of the mandible, which is an innovative point. In addition, the present paper has the strength of presenting data about one cohort of patients belonging to a specific population. In this way, the study could have further knowledge in this specific field. About the clinical relevance, we want to highlight that it is absolutely true that the rate of reported complications in this area is low, but the consequences could be extremely relevant, being among the few causes of death for such interventions. The paper wants to stress the importance to evaluate also such anatomic structures during treatment planning, potentially being as important as other anatomical structures that are commonly considered. Moreover, the evolution of implant techniques, such as the all on four technique, has in recent years drastically increased the number of implants placed in the mandibular symphysis, making this anatomical area one of the most interesting in terms of rehabilitation.

Table 3 Number of LF reported in different CT and cadaveric studies

\begin{tabular}{llll}
\hline Study & Country & Sample & LF \\
\hline Our study & Italy & 300 Patients, CBCT & 592 Midline, 283 lateral \\
Von Arx et al. [18] & Switzerland & 179 Patients, CBCT & 86 Midline, 131 lateral \\
Trost et al. [25] & Germany & 460 Patients, CT & 613 Midline, 231 lateral \\
Sheikhi et al. [19] & Turkey & 102 Patients, CBCT & 205 Midline \\
Sekerci et al. [11] & Turkey & 500 Patients, CBCT & 476 Midline \\
Liang et al. [20] & Belgium & 555 Patients, CT & 132 Midline \\
He et al. [14] & China & 200 Patients, CBCT & 683 \\
Rosano et al. [5] & Italy & 60 Cadaveric mandibles & 118 \\
Vandewalle et al. [3] & Belgium & 354 Dry mandibles & 347 \\
\hline
\end{tabular}


Anatomical knowledge of this area also affects other surgical procedures such as biopsies of the oral floor, maxillofacial oncological surgery and emergency surgery for traumas or injuries occurring in the area of the mandibular symphysis. The anatomical variables highlighted in this study lay the foundations for complex diagnoses at the vascular level of the lower jaw in the case of haemorrhages, and are essential for correctly directing emergency triage.

Some limitations of our study should be considered. First, the relatively small sample size of our series, indeed, we cannot exclude that a larger study population would have allowed to obtain a more powerful statistical analysis, even reaching interesting association of the number and anatomic distribution of LF with mandible measurements. Second, we did not evaluate the distance of LF from the alveolar ridge and tooth apex that, in turn, could be an important pre-operative finding to be evaluated before proceeding with implant procedures. Third, the methodology that we used to estimate the mandibular width has not been previously validated, but allowed us to investigate the correlation between the distribution of LF and mandible width. However, it is not felt that this fact was a real limitation, but rather something that deserves further investigation. Last, our CBCT analysis is based on the detection of bone canals, rather than the direct visualization of the vessels. The differences in the number and distribution of LF with some previous studies might be partly related to the different approaches used to assess the LF, for instance through cadaveric skulls which might enable to demonstrate a higher number of LF than CBCT studies. The limited, albeit high, spatial resolution of $\mathrm{CT}$ probably might be responsible for a lower detection rate of the LF, although according to several authors CBCT provides highly accurate data concerning mandible anatomy and state that the different frequencies reported in literature is mostly related to the anatomical variability related to different geographical regions. For instance, Rosano et al. [5] found LF in $100 \%$ of cases in their cadaveric study, while Tagaya et al. [21] published a double study on five cadavers and 200 patients using CBCT reporting the occurrence of LF in all cadavers and in $95 \%$ of patients.

\section{Conclusions}

In conclusion, we have shown the high variability of number and anatomic distribution of midline and lateral LF in a Caucasian Italian group of patients subjected to CBCT without reporting any association with gender and mandible width, shape, and bone thickness. The anatomical variability of the vascular bundles of the floor of mouth must be considered when dealing with surgery in the mandibular region included between the mental foramina to avoid dangerous and life-threating bleeding accidents.

\section{Abbreviations}

LF: Lingual foramina; CT: Computed tomography; CBCT: Cone-beam CT.

\section{Acknowledgements \\ None.}

\section{Authors' contributions}

Guarantor of integrity of the entire study: Silvio Taschieri; Study concepts: Silvio Taschieri, Stefano Corbella, Domenico Albano; Study design: Silvio Taschieri, Stefano Corbella, Luca Francetti; Data analysis and interpretation: Amel Silnovic, Carmelo Messina Statistical analysis: Luca Maria Sconfienza; Manuscript preparation: Domenico Albano; Manuscript editing: Silvio Taschieri, Stefano Corbella, Luca Maria Sconfienza; Manuscript review: Domenico Albano, Luca Francetti, Carmelo Messina. All authors read and approved the final manuscript.

\section{Funding}

This research did not receive any specific grant from funding agencies in the public, commercial, or not-for-profit sectors.

\section{Availability of data and materials}

All data are fully available upon reasonable request. The corresponding author should be contacted if someone wants to request the data.

\section{Declarations}

Ethics approval and consent to participate

This study was approved by our Institutional review board (Ospedale San Raffaele, Milano, Italy). All procedures were in accordance with the ethical standards of the responsible committee on human experimentation (institutional and national) and with the Helsinki Declaration of 1975, as revised in 2008.

\section{Consent for publication}

All individuals involved in this study provided written consent to use their imaging data for research purposes.

\section{Competing interests}

The authors declare that they have no competing interests.

\section{Author details}

${ }^{1}$ Department of Biomedical, Surgical and Dental Sciences, Università Degli Studi Di Milano, Milan, Italy. ${ }^{2}$ RCCS Istituto Ortopedico Galeazzi, Milan, Italy. ${ }^{3}$ Department of Oral Surgery, Institute of Dentistry, I. M. Sechenov First Moscow State Medical University, Moscow, Russia. ${ }^{4}$ Dipartimento Di Scienze Biomediche Per La Salute, Università Degli Studi Di Milano, Milan, Italy. ${ }^{5}$ Sezione Di Scienze Radiologiche, Dipartimento Di Biomedicina, Neuroscienze E Diagnostica Avanzata, Università Degli Studi Di Palermo, Palermo, Italy.

Received: 7 August 2021 Accepted: 4 January 2022

Published online: 20 January 2022

\section{References}

1. Nakajima K, Tagaya A, Otonari-Yamamoto M, Seki K, Araki K, Sano T, et al. Composition of the blood supply in the sublingual and submandibular spaces and its relationship to the lateral lingual foramen of the mandible. Oral Surg Oral Med Oral Pathol Oral Radiol. 2014;117. doi:https://doi.org/ 10.1016/j.oooo.2012.03.032.

2. Tang M, Ding M, Almutairi K, Morris SF. Three-dimensional angiography of the submental artery perforator flap. J Plast Reconstr Aesthetic Surg. 2011;64:608-13. https://doi.org/10.1016/j.bjps.2010.08.040.

3. Vandewalle G, Liang X, Jacobs R, Lambrichts I. Macroanatomic and radiologic characteristics of the superior genial spinal foramen and its bony canal. 2005 
4. Katsumi Y, Tanaka R, Hayashi T, Koga T, Takagi R, Ohshima H. Variation in arterial supply to the floor of the mouth and assessment of relative hemorrhage risk in implant surgery. Clin Oral Implants Res. 2013;24:434-40. https://doi.org/10.1111/j.1600-0501.2011.02348.x.

5. Rosano G, Taschieri S, Gaudy JF, Testori T, Del Fabbro M. Anatomic assessment of the anterior mandible and relative hemorrhage risk in implant dentistry: A cadaveric study. Clin Oral Implants Res. 2009;20:791-5. https://doi.org/10.1111/j.1600-0501.2009.01713.x.

6. Del Castillo-Pardo de Vera JL, López-Arcas Calleja JM, Burgueño-García M. Hematoma of the floor of the mouth and airway obstruction during mandibular dental implant placement: a case report. Oral Maxillofac Surg. 2008;12:223-6. doi:https://doi.org/10.1007/S10006-008-0134-4.

7. Kalpidis CDR, Setayesh RM. Hemorrhaging associated with endosseous implant placement in the anterior mandible: a review of the literature. J Periodontol. 2004;75:631-45. https://doi.org/10.1902/JOP.2004.75.5.631.

8. Blanc O, Krasovsky A, Shilo D, Capucha T, Rachmiel A. A life-threatening floor of the mouth hematoma secondary to explantation attempt in the anterior mandible. Quintessence Int. 2021;52:66-71. https://doi.org/10. 3290/J.QI.A45265

9. Taschieri S, Vitelli C, Albano D, Sconfienza LM, Del Fabbro M, Francetti $L$, et al. Evaluation of mental foramen and inferior alveolar nerve canal and its relationship to adjacent anatomical landmarks using cone-beam computer tomography. J Biol Regul Homeost AGENTS. 2020;35:107-15.

10. Bernardi S, Bianchi S, Continenza MA, Macchiarelli G. Frequency and anatomical features of the mandibular lingual foramina: systematic review and meta-analysis. Surg Radiol Anat. 2017;39:1349-57. https://doi.org/10. 1007/s00276-017-1888-X.

11. Sekerci $A E$, Sisman $Y$, Payveren MA. Evaluation of location and dimensions of mandibular lingual foramina using cone-beam computed tomography. Surg Radiol Anat. 2014;36:857-64. https://doi.org/10.1007/ s00276-014-1311-9.

12. Demiralp KO, Bayrak S, Orhan M, Alan A, Kursun-Cakmak ES, Orhan K. Anatomical characteristics of the lingual foramen in ancient skulls: A cone beam computed tomography study in an Anatolian population. Folia Morphol. 2018;77:514-20. https://doi.org/10.5603/FM.a2018.0009.

13. Butura CC, Galindo DF, Cottam J, Adams M, Jensen O. Hourglass mandibular anatomic variant incidence and treatment considerations for all-on-four implant therapy: report of 10 cases. J Oral Maxillofac Surg. 2011;69:2135-43. https://doi.org/10.1016/J.JOMS.2011.02.070.

14. He X, Jiang J, Cai W, Pan Y, Yang Y, Zhu K, et al. Assessment of the appearance, location and morphology of mandibular lingual foramina using cone beam computed tomography. Int Dent J. 2016;66:272-9. https:// doi.org/10.1111/idj.12242.

15. Patil S, Matsuda Y, Okano T. Accessory mandibular foramina: a CT study of 300 cases. Surg Radiol Anat. 2013;35:323-30. https://doi.org/10.1007/ s00276-012-1044-6.

16. Yildirim YD, Güncü GN, Galindo-Moreno P, Velasco-Torres M, Juodzbalys G, Kubilius M, et al. Evaluation of mandibular lingual foramina related to dental implant treatment with computerized tomography: a multicenter clinical study. Implant Dent. 2014;23:57-63. https://doi.org/10.1097/ID. 0000000000000012

17. Wang YM, Ju YR, Pan WL, Chan CP. Evaluation of location and dimensions of mandibular lingual canals: a cone beam computed tomography study. Int J Oral Maxillofac Surg. 2015;44:1197-203. https://doi.org/10.1016/j. ijom.2015.03.014.

18. Von Arx T, Matter D, Buser D, Bornstein MM. Evaluation of location and dimensions of lingual foramina using limited cone-beam computed tomography. J Oral Maxillofac Surg. 2011;69:2777-85. https://doi.org/10. 1016/j.joms.2011.06.198.

19. Sheikhi M, Mosavat F, Ahmadi A. Assessing the anatomical variations of lingual foramen and its bony canals with CBCT taken from 102 patients in Isfahan. Dent Res J (Isfahan). 2012;9(Suppl 1):S45-51. https://doi.org/10. 4103/1735-3327.107936.

20. Liang $X$, Jacobs $R$, Lambrichts I, Vandewalle $G$. Lingual foramina on the mandibular midline revisited: a macroanatomical study. Clin Anat. 2007:20:246-51. https://doi.org/10.1002/ca.20357.

21. Tagaya A, Matsuda Y, Nakajima K, Seki K, Okano T. Assessment of the blood supply to the lingual surface of the mandible for reduction of bleeding during implant surgery. Clin Oral Implants Res. 2009;20:351-5. https://doi.org/10.1111/j.1600-0501.2008.01668.x.
22. Soto R, Concha G, Pardo S, Cáceres F. Determination of presence and morphometry of lingual foramina and canals in Chilean mandibles using cone-beam CT images. Surg Radiol Anat. 2018;40:1405-10. https://doi. org/10.1007/s00276-018-2080-7.

23. Przystańska A, Bruska M. Anatomical classification of accessory foramina in human mandibles of adults, infants, and fetuses. Anat Sci Int. 2012;87:141-9. https://doi.org/10.1007/s12565-012-0136-z.

24. Xie L, Li T, Chen J, Yin D, Wang W, Xie Z. Cone-beam CT assessment of implant-related anatomy landmarks of the anterior mandible in a Chinese population. Surg Radiol Anat. 2019;41:927-34. https://doi.org/10.1007/ s00276-019-02250-7.

25. Trost M, Mundt T, Biffar R, Heinemann F. The lingual foramina, a potential risk in oral surgery. A retrospective analysis of location and anatomic variability. Ann Anat. 2020;231. doi:https://doi.org/10.1016/J.AANAT.2020. 151515.

\section{Publisher's Note}

Springer Nature remains neutral with regard to jurisdictional claims in published maps and institutional affiliations.

Ready to submit your research? Choose BMC and benefit from

- fast, convenient online submission

- thorough peer review by experienced researchers in your field

- rapid publication on acceptance

- support for research data, including large and complex data types

- gold Open Access which fosters wider collaboration and increased citations

- maximum visibility for your research: over $100 \mathrm{M}$ website views per year

At BMC, research is always in progress.

Learn more biomedcentral.com/submissions 